\title{
European cobalt sources identified in the production of Chinese famille rose porcelain
}

\author{
Rita Giannini ${ }^{1}$, Ian C. Freestone ${ }^{2}$, Andrew J. Shortland ${ }^{1}$ \\ ${ }^{1}$ Cranfield Forensic Institute \\ Cranfield University \\ Defence Academy of the United Kingdom \\ Shrivenham \\ Wilts \\ SN6 8LA \\ a.shortland@cranfield.ac.uk \\ 2 Institute of Archaeology \\ UCL \\ 31-34 Gordon Square \\ London WC1H 0PY
}

\section{Abstract}

The blue pigments on 112 fragments or small objects of Qing Dynasty Chinese, 95 of underglaze blue and white and 17 overglaze enamelled porcelains were analysed by LA-ICPMS. The underglaze blues on both blue and white and polychrome objects were created with a cobalt pigment that was rich in manganese with lesser nickel and zinc. This suite of accessory elements is generally considered to be characteristic of local, Chinese, sources of pigments. However, the blue enamels were very different. The cobalt pigment here has low levels of manganese and instead is rich in nickel, zinc, arsenic and bismuth. No Chinese source of cobalt with these characteristics is known, but they closely match the elements found in the contemporary cobalt source at Erzgebirge in Germany. Textual evidence has been interpreted to suggest that some enamel pigment technologies were transferred from Europe to China, but this is the first analytical evidence to be found that an enamel pigment itself was imported. It is possible that this pigment was imported in the form of cobalt coloured glass, or smalt, which might account for its use in enamels, but not in an underglaze, where the colour might be susceptible to running. Furthermore, the European cobalt would have given a purer shade of blue than the manganese-rich Chinese cobalt.

\section{Keywords}

CHINA, PORCELAIN, COBALT BLUE, FAMILLE ROSE, LA-ICPMS 


\section{Introduction}

Cobalt is a strong colorant used in many areas of the world for the production of pigments and blue glass and glazes. Its earliest use is probably in Egypt in the Late Bronze Age, around 16 th century BC. Its first use in China was in the Spring and Autumn period (770-475 BC) when it was used as a colouring agent in glazed beads then later in low-firing glazes on Tang sancai and blue glazed earthenwares (Garner 1957, p.1). Its first use in Chinese glass dates back to the Han Dynasty, while the earliest Chinese example of the use of cobalt as an underglaze pigment comes from the ninth century, Tang port of Yangzhou City (Wang et al. 1993, see also Wood et al. 2007). It was perfected in the blue and white porcelain of the Yuan dynasty in the early fourteenth century $\mathrm{CE}$, and the technology was adopted at Jingdezhen, which went on to become the most important kiln site in China, effectively a city devoted to the production of porcelain (Tichane 1983; Harrison-Hall 1997). Chinese blue and white porcelain represents one of the most successful and influential developments in the history of ceramic technology. A convergence of the technologies of high-fired white stoneware and underglaze painting with a cobalt pigment, it became a major component of Chinese porcelain production and was particularly important as an export ware, initially to the Islamic world and later to Europe (Medley, 1989, p. 178). It has been emulated by industries across the world, and remains commercially important today (Finlay 1998). Here new data are reported which demonstrate for the first time that European cobalt sources played an important role in the development of Chinese enamelled porcelains in the eighteenth century.

Cobalt has attracted archaeometric attention because the relatively limited number of sources that were accessible to early craftsmen, along with the variable compositions of the ores, makes it possible to characterise and attribute the pigments to their region or even mine of origin (Gratuze 2013). In particular, the clear interplay of style and technology between the blue and white wares of China and the Near East from the Tang to Ming periods (7th-17th centuries; (Medley, 1989; Rawson, 1984; Vainker, 1989)) has led to increasingly sophisticated analytical studies with a view to determining the source of cobalt and contributing to an understanding of the processes of technological transfer and innovation (Kerr and Wood, 2004; Wen and Pollard, 2016; e.g. Wen et al., 2007; Zhu et al., 2015).

The application of low-firing lead-rich coloured enamels over the glaze of previously high-fired stonewares and porcelains can be traced back to the end of the 12th to the beginning of the 13th Century (Medley 1989; Wood 1999; Kerr and Wood 2004). In China they were first applied onto white slipped high-firing, glazed stonewares, known as cizhou wares, and in the late 14th century the enamelling techniques used in the northern cizhou kilns spread to Jingdezhen in the south (Wood, 1999). In the Qing dynasty (1644-1911) in the reign of the the Kangxi emperor (1672-1722) the initial palette of the famille verte family, known in Chinese as wucai or five-colour was developed, comprising copper-green, iron-yellow, iron-red and turquoise overglaze enamels on porcelain decorated with underglaze cobalt (e.g. Medley, 1989; Vainker, 1989, p. 202). 
The Kangxi reign (1662-1722) was a period of great stability and support for the craft

Fig. 1 Detail of the Famille rose enamels painted in this case on the Daoguang-reign period porcelain (a) B.fr.1850.2 and (b)B.fr.1850.3 (b). (photo: R Giannini).

a)
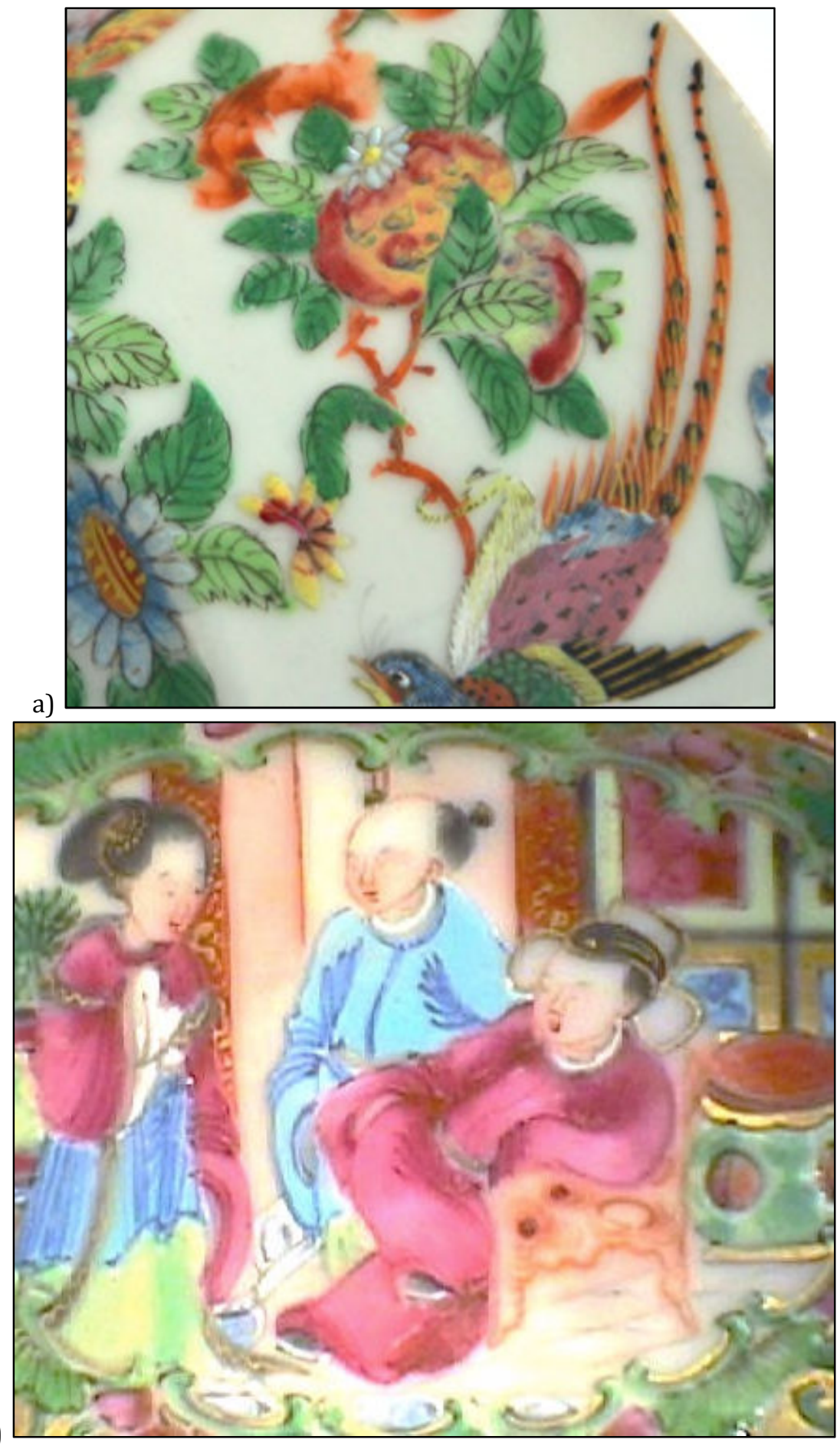

industries, including an emphasis on painted enamel work on glass and metal, as well as ceramic, which was driven by the emperor himself. Workshops were attached to the Imperial Court in Beijing and foreign craftsmen were sought to 
develop techniques. Late in the seventeenth century the mature famille verte palette, including for the first time an overglaze cobalt blue enamel, was developed (Vainker, 1989). Towards the very end of the Kangxi period (Sato, 1981; Vainker, 1989), extensive development work in the Palace workshops in Beijing, discussed in detail by Kerr and Wood (2004) and also by Curtis (2009) led to the development of the famille rose group of enamels, which included a red based upon colloidal gold and an arsenic opaque white, which in mixing could produce a wide range of red and pink shades (Fig. 1). This palette appears to have been transferred for production at the Imperial kilns in Jingdezhen at the beginning of the reign of the succeeding emperor, Yongzheng (1722-1735). In addition to the high artistic quality of some of the ceramics, several characteristics of famille rose or fencai have attracted scholarly attention. The development of a gold-based pink at this time corresponds with the development of gold ruby glass in Europe, the practical application of which is particularly associated with the German chemist Johann Kunckel (Hunt, 1976). Europeans with knowledge and skills in glass and enamel production were attached to the Chinese Imperial Court and workshops were established, for example the glass workshop headed by the Jesuit missionary Kilian Stumpf in 1697 (Curtis, 1993). Furthermore, one of the terms by which the famille rose palette was known to the Chinese craftsmen was "foreign colours" (yangcai), and the official list of porcelain produced at Jingdezhen in the Yongzheng reign refers to the use of European or foreign decoration on at least six occasions (Bushell, 1896). All of this led to the idea that the famille rose palette was heavily influenced by European practice and possibly that the technology itself was transferred. However, limited analytical work has so far failed to identify any unambiguously European compositions on famille rose pieces (Kingery and Vandiver, 1986) and furthermore has suggested a strong link with compositions of earlier Chinese cloisonné enamels on metalwork (Henderson, 1989; Kerr and Wood, 2004; Mills and Kerr, 1999; Vainker, 1989). The influences on the development of famille rose therefore continue to be a subject of significant interest.

Our understanding of porcelain production and technology in the Qing Dynasty has been surprisingly dependent upon the account of a single person, Père d'Entrecolles, a French Jesuit missionary. Through conversations with the craftsmen and direct observation, d'Entrecolles was able to document many aspects of industrial practice, in two famous letters dated 1712 and 1722, which attracted wide attention in the eighteenth century as Europeans attempted to discover the secret of porcelain. English translations in print are provided by Burton (1906; slightly abridged) and Tichane (1983) with an on-line version provided at www.gutenberg.com. Significantly for the present work, d'Entrecolles's last communication from Jingdezhen was more-or-less at the time when famille rose production was introduced at Jingdezhen, but he does make some interesting observations about earlier enamels and cobalt.

Cobalt blue was such a widespread colour in Chinese ceramic production from the fourteenth century onwards that a critical role in the development of the enamel palette has not been considered in detail. However, there are some tantalising indications that production of a cobalt blue enamel was not straightforward. Firstly, there is the fact that it was the very last of the overglaze colours to be added to the earlier famille verte palette (Vainker, 1989, p. 202). Secondly, the analysis of famille 
verte enamels by Kingery and Vandiver (1986) reveals the cobalt blue to be the only colour with an elevated potash content of around $6 \%$ relative to less than $0.5 \%$ for the other colours. With some hindsight, this suggests a deliberate addition of potassium to the blue and a significant difference in the technology of the base glass relative to the other colours at that time.

The present paper reports new quantitative results for the cobalt on the later blueand-white ceramics of the Qing Dynasty (1644-1912). While most are agreed that by the end of Ming times the pigments used on Chinese underglaze blue were obtained from Chinese sources (Wen and Pollard, 2016; Wen et al., 2007; Zhu et al., 2015), our results suggest that the situation under the Qing was more complex. In particular, we focus on the pigments on the polychrome enamelled wares which were extensively exported to Europe during this period.

\subsection{Cobalt pigments}

The blue colour produced by cobalt-based pigments can be due to the presence of cobalt in both its crystalline and solution-ionic forms. As the $\mathrm{Co}^{3+}$ ion is not stable in the temperature range required for glass melting, only those cobalt compounds which are derived from the divalent cobalt ion $\mathrm{Co}^{2+}$ are of interest in glass technology (metallic Co assumes a significant role only in the field of enamel on metal, where it contributes to the adherence of the ground coats) (Weyl, 1951, p. 170). In particular, in alkaline glazes, $\mathrm{Co}^{2+}$ ions in tetrahedral coordination (i.e. present in the vitreous structure as glass formers, in the form of $\mathrm{CoO}_{4}$ complexes) give rise to blues or blue-purples (or blue hues in lead-based matrices), while in the octahedral coordination $\left(\mathrm{Co}^{2+}\right.$ ions are inserted in the position occupied by alkali ions, $\mathrm{CoO}_{6}$ complexes) confer pink hues to the glass (Weyl, 1951, pp. 179-80, 182-4). Cobalt is one of the most stable and powerful colouring agents and saturated blue tints in common glassy systems occur for $\mathrm{CoO}$ concentrations as low as $0.25 \%$ (noticeable blues are already observed at levels of c. $0.005 \%$ CoO) (Kerr et al., 2004; Weyl, 1951, pp. 179-80). In Chinese blue underglazes $\mathrm{CoO}$ is usually found at levels of about $0.1-1 \%$ (Kerr et al., 2004).

Cobalt does not exist as a native metal, though there are many cobalt-bearing minerals from which it can be extracted (Henderson, 2000, p. 30). The analysis of the impurities naturally occurring in the cobalt ores (e.g. iron, copper, manganese, nickel, arsenic, sulphur, bismuth) might therefore provide a valuable support in revealing the cobalt sources employed by the ancient craftsmen. For example, the association of arsenic and sulphur (and sometimes zinc) may suggest the use of cobaltite (CoAsS) or smaltite ( $\mathrm{CoAs} 2)$, while nickel and arsenic of the minerals erythrite $\left(\mathrm{Co}_{3}\left(\mathrm{AsO}_{4}\right)_{2} 8 \mathrm{H}_{2} \mathrm{O}\right)$ or skutterudite $((\mathrm{Co}, \mathrm{Ni}) \mathrm{As} 3-\mathrm{x})$, manganese of the mineral asbolane $(\mathrm{Co}, \mathrm{Ni})_{1-y}\left(\mathrm{MnO}_{2}\right)_{2-\mathrm{x}}(\mathrm{OH})_{2-2 \mathrm{y}+2 \mathrm{x}} \mathrm{n}\left(\mathrm{H}_{2} \mathrm{O}\right)$. Several cobalt-compounds can also contain significant amount copper. Finally, blue compounds could also be obtained from cobalt, nickel, iron and copper-rich residues after separating bismuth from its ores (Frank, 1982). 


\section{Methodology}

\subsection{Sample selection}

Several sets of porcelain samples were chosen for analysis. All fragments had to be small enough to fit into the sample chamber of the laser system, so less than 100x100x $25 \mathrm{~mm}$. The first set (codes N.bw.R* ${ }^{*}$ ) were blue and white jar lids excavated at Jingdezhen and lent by Professor Nigel Wood and Oxford University, all dated from $17^{\text {th }}$ to $20^{\text {th }}$ centuries $\mathrm{AD}$. The next set were sherds from either the Vung Tao Cargo (B.bw.VTC.1690-*) or the Nanking Cargo (B.bw.NC.1750-*), dated by Mary Tregear (Ashmolean Museum) to 1690 and 1750 or thereabouts. Most of this was Jingdezhen export porcelain bound for the European market. The next set was from the Victoria and Albert Museum sherd collections and represent a variety of fragments from the Qing Dynasties including blue and white (V\&M.bw.*), famille rose (V\&M.fr.*) and a single famille vert (V\&M.fv.1385-1902). The final set were acquired from private collections and represent blue and white (B.bw.[date].*), famille rose (B.fr.[date].*) and a single famille verte (B.fv.1700.1) fragment of various porcelain types dating to the $18^{\text {th }}$ and $19^{\text {th }}$ centuries. In total the blue areas on 92 examples of blue and white underglaze, three underglazed blue on a polychrome vessels and 17 famille rose blue enamels were analysed.

\subsection{LA-ICPMS}

Table 1: LA-ICPMS operating conditions

\begin{tabular}{|c|c|}
\hline ICP-Q-MS - Thermo Electron Corporation XSERIES 2 & Working Conditions \\
\hline RF power (W) & $1430-1470$ \\
\hline Coolant gas flow rate $\left(\mathrm{L} \mathrm{min}^{-1}\right)$ & $15(\mathrm{Ar})$ \\
\hline Auxiliary gas glow rate $\left(\mathrm{L} \mathrm{min}^{-1}\right)$ & 0.9 \\
\hline Nebulizer flow rate $\left(\mathrm{L} \mathrm{min}^{-1}\right)$ & $0.8-1.2$ \\
\hline Extraction (V) & $-720 /-750$ \\
\hline Detector mode & counting and analogue mode \\
\hline Acquisition mode & peak hopping \\
\hline Channel per mass & 1 \\
\hline Channel spacing & 0.02 \\
\hline Dwell time (ms) & $20-50$ \\
\hline Sweeps & $15-20$ \\
\hline Total acquisition time (s) & $50-60$ \\
\hline Sampling events & $1-3$ \\
\hline Replicate per sample & $>3$ \\
\hline $\mathrm{ThO}^{+} / \mathrm{Th}^{+}$ & $<0.02 \%$ \\
\hline $\mathrm{CeO}^{+} / \mathrm{Ce}^{+}$ & $<0.2 \%$ \\
\hline LA - New Wave Research, Q switched Nd:YAG & Working Conditions \\
\hline Wavelength $(\mathrm{nm})$ & 213 \\
\hline Laser ablation chamber & Standard \\
\hline $\begin{array}{l}\text { Ablation mode } \\
\text { spot diameter }(\mu \mathrm{m})\end{array}$ & $\begin{array}{l}\text { spot - scan }\left(<1500 \mu \mathrm{m} \text { path, } 10 \mu \mathrm{m} \mathrm{s}^{-1}\right) \\
80\end{array}$ \\
\hline Pulse time (ns) & 2 \\
\hline Energy (m) & 0.42 \\
\hline Energy density (fluence) $\left(\mathrm{J} \mathrm{cm}^{-2}\right)$ & $>20$ \\
\hline Pulse repetition rate $(\mathrm{Hz})$ & 10 \\
\hline Carrier gas flow rate $\left(\mathrm{ml} \mathrm{min}^{-1}\right)$ & $500(\mathrm{He})$ \\
\hline
\end{tabular}

The sherds were ablated directly in the large sample chamber of a New Wave 213 laser attached to a Thermo Series II ICPMS. The ablation conditions and ICPMS set up were optomised using a series of experimental samples and the conditions used are shown in Table 1. The results of runs on the unknowns were interspersed with gas blank runs and calibrated against NIST SRM 610 glass reference material, doped with a nominal concentration of $500 \mathrm{ppm}$ for most trace elements, and NIST 612 (50 ppm nominal concentration) using the 
consensus values (Jochum et al., 2011). Each batch of samples included multiple measurements from NIST 610 and 612 throughout the duration of the session to allow for correction of instrument drift. Repeat measurements of Corning A were made throughout the analytical period and the results of those analyses are reported in Table 2 and compared to accepted values (Shortland et al., 2007; Vicenzi et al., 2002; Wagner et al., 2012). The results reveal that for the majority of trace elements agreement with accepted values, as expressed by the percentage difference between the determined and accepted values (RD) is usually better than $20 \%$. The greatest deviation from accepted or consensus values was shown by $\mathrm{P}$ and $\mathrm{K}$, regarded as difficult elements to determine by LAICPMS. Phosphorus has a high ionization potential and all isotopes of potassium have high background counts because of their proximity to Ar, the plasma gas.

Table 2:Runs by LA-ICPMS against Corning A secondary standard showing deviation from accepted values.

\begin{tabular}{|c|c|c|c|c|c|c|}
\hline & \multicolumn{6}{|c|}{ CMG A } \\
\hline Analyte & $\begin{array}{l}\text { Measured } \\
(\mathrm{n}=33,11 \\
\text { runs })\end{array}$ & SD & $\begin{array}{l}\text { Accepted } \\
\text { value }^{1,2}\end{array}$ & $\begin{array}{c}\text { Accepted } \\
\text { value }^{3}\end{array}$ & $\mathrm{RD}^{1,2}$ & $\mathrm{RD}^{3}$ \\
\hline & $\mathrm{mg} \cdot \mathrm{kg}^{-1}$ & $\mathrm{mg} \cdot \mathrm{kg}^{-1}$ & $\mathrm{mg} \cdot \mathrm{kg}^{-1}$ & $\mathrm{mg} \cdot \mathrm{kg}^{-1}$ & $\%$ & $\%$ \\
\hline Li & 45 & 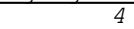 & 46 & 51 & -2 & -12 \\
\hline $\mathrm{Be}$ & 0.06 & 0.03 & 0.06 & & 0.4 & \\
\hline B & 607 & 54 & 537 & 851 & 15 & -28 \\
\hline $\mathrm{Na}$ & 102171 & 4178 & 106083 & 99407 & -3 & 3 \\
\hline $\mathrm{Mg}$ & 13332 & 802 & 16043 & 15078 & -17 & -12 \\
\hline Al & 4962 & 118 & 5291 & 4339 & -6 & 14 \\
\hline $\mathrm{Si}$ & 317805 & 3172 & $31 \overline{0883}$ & 316768 & 2 & 0.3 \\
\hline P & 655 & 35 & 341 & 371 & 92 & 77 \\
\hline $\mathrm{K}$ & 24152 & 1449 & 22639 & 28714 & 7 & -16 \\
\hline $\mathrm{Ca}$ & 35626 & 788 & 35954 & 35311 & -0.5 & 0.9 \\
\hline $\mathrm{Ti}$ & 4697 & 119 & 4226 & 4428 & 11 & 6 \\
\hline V & 37 & 770 & 34 & 39 & 0.6 & -12 \\
\hline $\mathrm{Cr}$ & 20 & 0.6 & 18 & 21 & 10 & -4 \\
\hline $\mathrm{Mn}$ & 7594 & 312 & 6921 & 8752 & 10 & -13 \\
\hline $\mathrm{Fe}$ & 7171 & 442 & 6537 & 6841 & 10 & 5 \\
\hline Co & 1222 & 27 & 1188 & 1336 & 3 & -9 \\
\hline $\mathrm{Ni}$ & 184 & 5 & 160 & 181 & 15 & 2 \\
\hline $\mathrm{Cu}$ & 9700 & 408 & 7842 & 8786 & 24 & 10 \\
\hline $\mathrm{Zn}$ & 461 & 42 & 410 & 386 & 14 & 20 \\
\hline As & 29 & 3 & 25 & & 15 & \\
\hline $\mathrm{Rb}$ & 80 & 8 & 82 & 82 & 0.02 & -0.9 \\
\hline $\mathrm{Sr}$ & 874 & 85 & 860 & 897 & 3 & -1 \\
\hline $\mathrm{Zr}$ & 41.7 & 0.8 & 40 & 37 & 4 & 13 \\
\hline $\mathrm{Nb}$ & 0.56 & 0.06 & 0.6 & & -5 & \\
\hline $\mathrm{Ag}$ & 16.3 & 0.4 & 14 & & 14 & \\
\hline $\mathrm{Sn}$ & 1526 & 25 & 1194 & 1357 & 28 & 12 \\
\hline $\mathrm{Sb}$ & 14849 & 1381 & 10649 & 14002 & 39 & 6 \\
\hline $\mathrm{Cs}$ & 0.29 & 0.04 & 0.2 & & 22 & \\
\hline $\mathrm{Ba}$ & 4444 & 94 & 3905 & 4122 & 10 & 8 \\
\hline $\mathrm{La}$ & 0.31 & 0.03 & 0.3 & & 12 & \\
\hline $\mathrm{Ce}$ & 0.23 & 0.03 & 0.2 & & -3 & \\
\hline $\mathrm{Au}$ & 0.11 & 0.02 & 0.1 & & 11 & \\
\hline $\mathrm{Pb}$ & 699 & 15 & 595 & 678 & 18 & 3 \\
\hline $\mathrm{Bi}$ & 9.5 & 0.3 & 7.8 & 9.0 & 22 & 5 \\
\hline Th & 0.31 & 0.02 & 0.3 & & 3 & \\
\hline $\mathrm{U}$ & 0.18 & 0.02 & 0.2 & & 12 & \\
\hline
\end{tabular}

${ }^{1}$ Shortland et al., 2007

${ }^{2} \mathrm{Vicenzi}$ et al., 2002

${ }^{3}$ Wagner et al., 2012

The results were calibrated using the mathematical approach first proposed by Gratuze (Gratuze, 1999) as an alternative to the use of an internal standard. The protocol used here followed that laid out by van Elteren (van Elteren et al., 2009). This essentially works in a similar fashion to a normalised EDS system on an SEM. It assumes that all elements are measured and calculates oxygen by stoichiometry. The total is then normalised to $100 \%$ and either presented as weight percent oxide or converted back to elemental ppm. 


\section{Results}

Table 3: Averages of undecorated glazes for blue and white and polychrome wares

\begin{tabular}{lrrrrrrrrr}
\hline & Co & $\mathrm{Mn}$ & $\mathrm{Ni}$ & $\mathrm{Cu}$ & $\mathrm{Zn}$ & $\mathrm{As}$ & $\mathrm{Ba}$ & $\mathrm{Bi}$ & $\mathrm{U}$ \\
\cline { 2 - 9 } $\begin{array}{l}\text { Qing A } \\
\text { Average * }\end{array}$ & 37.7 & 988 & 31.6 & 46.5 & 54.9 & 2.1 & 79.4 & 0.1 & 11.2 \\
SD & 64.6 & 1177 & 34.1 & 24.7 & 28.6 & 1.5 & 36.5 & 0.1 & 2.5 \\
& & & & & & & & & \\
Qing B & & & & & & & & & \\
Average ** & 72.9 & 461 & 48.4 & 39.5 & 29.4 & 1.7 & 94.6 & 0.0 & 10.1 \\
SD & 60.2 & 100 & 72.4 & 11.7 & 17.0 & 1.7 & 30.3 & 0.0 & 1.8 \\
& & & & & & & & & \\
Qing C & & & & & & & & & \\
Average & 8.5 & 873 & 3.5 & 27.6 & 54.2 & 2.3 & 720 & 0.0 & 3.1 \\
SD & 5.2 & 577 & 1.0 & 30.6 & 34.2 & 3.5 & 188 & 0.0 & 0.9 \\
& & & & & & & & & \\
Polychrome & 4.3 & 721 & 21.9 & 99.0 & 65.1 & 5.1 & 78.7 & 0.6 & 11.9 \\
Average & 5.3 & 276 & 12.0 & 118 & 139 & 14.7 & 28.9 & 2.0 & 3.2
\end{tabular}

The LA-ICPMS was used to provide analyses of the undecorated glazes on the blue and white and polychrome (enamelled) wares (Table 3) and the areas of dark-blue glaze in the underglazed blue and white (Table 4), the dark blue underglaze in the polychrome (Table 4) and enamels in the polychrome famille verte and famille rose (Table 5). Care was taken to target the darkest blues and of as similar a hue as possible, to minimise possible differential diffusion of colouring wlements in the glaze. The major and minor element characteristics of the glazes are well known (Wood, 1999), and have been extensively published and our detailed analyses of these and the new trace element data will be presented and discussed elsewhere (Giannini et al, in prep). However, the results are pertinent to the present study of cobalt blue. The blue and white wares can be split into three slightly differing groups, Qing A, B and C. The largest group, Qing A, is the most similar to the polychrome wares. Both Qing A and the polychrome wares are consistent with production at Jingdezhen, as would be expected, whereas Qing B and Qing C are probably from other production sites (Giannini et al, in prep). Table 3 presents the analyses of undecorated white glazes, showing those elements that are conventionally thought to be linked with cobalt colorants (as discussed above). The table shows that the white glazes themselves have low levels of these elements, typically tens of ppm or lower for most. The exceptions are manganese in all the porcelain analysed, where the average content is up to 1,000 ppm $\mathrm{Mn}$ and $\mathrm{Ba}$ in the Qing $\mathrm{C}$ group, which averages $720 \mathrm{ppm} \mathrm{Ba}$. This shows that with the exception of these two elements (discussed below) the raw materials of the glazes do not significantly contribute to elevated concentrations of those elements associated with the blue colourants, which are the main interest of this paper. Even though the underglaze blue is 
analysed through the glaze and intimately mixed with it, the contribution of the overlying glaze in terms of these elements is minimal.

Table 4: LA-ICPMS analyses of underglaze blue areas of glazes on Qing blue and white Chinese porcelains.

$\begin{array}{lllllllllll}\text { Sample } & \text { Fe } & \text { Co } & \text { Mn } & \text { Ni } & \mathrm{Cu} & \mathrm{Zn} & \text { As } & \text { Ba } & \text { Bi } & \text { U }\end{array}$

QING Group 'A'

\begin{tabular}{|c|c|c|c|c|c|c|c|c|c|c|}
\hline N.bw.R1 & 7289 & 1934 & 12084 & 149 & 35 & 103 & 4.8 & 352 & 0 & 12.1 \\
\hline N.bw.R2 & 6609 & 1226 & 9028 & 85 & 44 & 73 & 6.2 & 212 & 0 & 11.1 \\
\hline N.bw.R60 & 6618 & 415 & 2626 & 16 & 43 & 42 & 5.7 & 105 & 0 & 11.4 \\
\hline N.bw.R76 & 6690 & 3768 & 13420 & 361 & 18 & 223 & 3.2 & 321 & 0.1 & 9.5 \\
\hline B.VTC.bw.1690.1 & 9680 & 11418 & 51876 & 780 & 63 & 272 & 72.2 & 934 & 0 & 10.2 \\
\hline V\&A.bw.C.459-1915 & 7986 & 3694 & 20520 & 105 & 59 & 172 & 113.3 & 229 & 0 & 9.3 \\
\hline V\&A.bw.C.94-1952 & 5038 & 605 & 4058 & 101 & 74 & 86 & 1.8 & 57 & 0.2 & 6 \\
\hline N.bw.R17 & 7985 & 3814 & 17586 & 259 & 31 & 153 & 248.2 & 337 & 0 & 10.5 \\
\hline N.bw.R31 & 7657 & 3034 & 20386 & 62 & 80 & 95 & 62.1 & 499 & 0 & 10.9 \\
\hline N.bw.R49 & 8170 & 3794 & 14242 & 197 & 37 & 107 & 36.1 & 289 & 0 & 10 \\
\hline N.bw.R59 & 6100 & 2335 & 13461 & 51 & 40 & 102 & 8.8 & 394 & 0.1 & 11.9 \\
\hline B.bw.1700.1 & 8413 & 3548 & 16287 & 189 & 26 & 50 & 62.3 & 366 & 0 & 9.2 \\
\hline B.bw.1720.1 & 5855 & 1698 & 10208 & 62 & 35 & 119 & 20.7 & 247 & 0 & 11.1 \\
\hline B.bw.1720.2 & 4630 & 609 & 3006 & 16 & 48 & 58 & 2.5 & 145 & 0.1 & 10.3 \\
\hline N.bw.R15 & 6089 & 4749 & 9966 & 186 & 36 & 117 & 62.7 & 201 & 0 & 9.3 \\
\hline N.bw.R18 & 8688 & 7742 & 23700 & 375 & 24 & 159 & 123 & 269 & 0 & 10.4 \\
\hline N.bw.R20 & 13767 & 4605 & 25987 & 502 & 43 & 121 & 634.5 & 477 & 0 & 13 \\
\hline N.bw.R21 & 7557 & 3055 & 11436 & 55 & 39 & 128 & 298.3 & 524 & 0 & 13.9 \\
\hline N.bw.R23 & 11679 & 6722 & 17944 & 129 & 28 & 207 & 12.3 & 351 & 0 & 9.3 \\
\hline N.bw.R24 & 6195 & 1892 & 11972 & 80 & 51 & 58 & 2.8 & 250 & 0 & 8.1 \\
\hline N.bw.R26 & 5936 & 1325 & 6495 & 41 & 24 & 79 & 11.4 & 147 & 0 & 18.3 \\
\hline N.bw.R28 & 6995 & 3922 & 12952 & 388 & 42 & 102 & 604 & 519 & 0.1 & 7.3 \\
\hline N.bw.R29 & 9712 & 4142 & 14214 & 46 & 24 & 87 & 8.5 & 271 & 0 & 10.4 \\
\hline N.bw.R32 & 5986 & 2854 & 14960 & 102 & 26 & 64 & 4.7 & 272 & 0 & 12.9 \\
\hline N.bw.R33 & 5585 & 1629 & 14918 & 111 & 52 & 76 & 17 & 318 & 0 & 15.9 \\
\hline N.bw.R34 & 8050 & 3370 & 12097 & 54 & 31 & 118 & 19.9 & 203 & 0 & 10.2 \\
\hline N.bw.R38 & 7298 & 2001 & 8061 & 49 & 40 & 92 & 73.4 & 246 & 0 & 10.7 \\
\hline N.bw.R40 & 7290 & 1903 & 12179 & 79 & 51 & 65 & 7.5 & 192 & 0 & 14 \\
\hline N.bw.R41 & 5631 & 1642 & 12821 & 79 & 34 & 51 & 2.4 & 348 & 0 & 10.7 \\
\hline N.bw.R42 & 7293 & 2997 & 17957 & 73 & 31 & 72 & 16.3 & 275 & 0 & 9.5 \\
\hline N.bw.R43 & 12191 & 4614 & 18144 & 159 & 44 & 146 & 148.3 & 441 & 0 & 9 \\
\hline N.bw.R45 & 5795 & 3480 & 7345 & 197 & 23 & 121 & 54.4 & 194 & 0 & 8.2 \\
\hline N.bw.R47 & 6782 & 1729 & 7447 & 83 & 30 & 74 & 8.2 & 247 & 0 & 9.2 \\
\hline N.bw.R48 & 5670 & 3040 & 14700 & 27 & 32 & 100 & 2.8 & 327 & 0 & 9.4 \\
\hline N.bw.R50 & 8095 & 1302 & 6125 & 17 & 38 & 64 & 39.3 & 178 & 0 & 8 \\
\hline N.bw.R51 & 6262 & 1394 & 9865 & 43 & 34 & 58 & 2.3 & 197 & 0 & 13 \\
\hline
\end{tabular}




\begin{tabular}{|c|c|c|c|c|c|c|c|c|c|c|}
\hline N.bw.R52 & 6918 & 3155 & 7589 & 203 & 35 & 153 & 14.1 & 184 & 0 & 11.7 \\
\hline N.bw.R53 & 9447 & 2095 & 13368 & 81 & 44 & 93 & 5.4 & 180 & 0.7 & 52.8 \\
\hline N.bw.R54 & 9444 & 2094 & 13422 & 94 & 47 & 120 & 4 & 199 & 0.7 & 12.5 \\
\hline N.bw.R55 & 7863 & 6463 & 40197 & 326 & 31 & 177 & 106.6 & 668 & 0 & 9.5 \\
\hline N.bw.R57 & 6357 & 698 & 5919 & 13 & 13 & 74 & 5.7 & 261 & 0 & 7 \\
\hline N.bw.R61 & 8556 & 459 & 4597 & 27 & 38 & 71 & 2.9 & 94 & 0.2 & 17 \\
\hline N.bw.R62 & 7298 & 1683 & 5852 & 38 & 27 & 69 & 5.8 & 164 & 0.1 & 9.4 \\
\hline N.bw.R72 & 9407 & 2238 & 9358 & 24 & 23 & 188 & 2.7 & 118 & 0 & 11.1 \\
\hline B.bw.1740.1 & 9725 & 3070 & 18225 & 106 & 52 & 123 & 2.3 & 316 & 0 & 9.6 \\
\hline B.bw.1750.1 & 8139 & 3969 & 18614 & 204 & 49 & 187 & 115 & 559 & 0 & 12.1 \\
\hline B.bw.1750.2 & 7654 & 2302 & 10252 & 28 & 122 & 86 & 4.7 & 245 & 0 & 6.3 \\
\hline B.NC.bw.1750.1 & 8113 & 2091 & 12259 & 87 & 42 & 95 & 54.9 & 319 & 0 & 13.5 \\
\hline B.NC.bw. 1750.2 & 7421 & 2296 & 11762 & 141 & 38 & 86 & 66 & 358 & 0 & 12 \\
\hline B.NC.bw. 1750.3 & 9217 & 4734 & 26102 & 102 & 35 & 178 & 39.7 & 686 & 0 & 9.3 \\
\hline B.NC.bw.1750.4a & 9653 & 2172 & 13682 & 59 & 46 & 78 & 4.2 & 438 & 0 & 11.4 \\
\hline B.NC.bw.1750.4b & 7788 & 1767 & 13400 & 51 & 47 & 116 & 22.6 & 282 & 0.1 & 11.1 \\
\hline B.NC.bw. 1750.5 & 9228 & 3237 & 18034 & 234 & 36 & 112 & 170.2 & 404 & 0 & 8 \\
\hline B.NC.bw.1750.6a & 6984 & 1001 & 6394 & 41 & 38 & 45 & 3.9 & 221 & 0 & 12 \\
\hline B.NC.bw.1750.6b & 7450 & 2067 & 11431 & 40 & 27 & 35 & 43.8 & 339 & 0 & 11.9 \\
\hline B.bw.1780.6 & 6984 & 2319 & 12131 & 242 & 75 & 80 & 119.3 & 545 & 0 & 8.2 \\
\hline N.bw.R16 & 4846 & 1158 & 10915 & 154 & 43 & 55 & 1.4 & 142 & 0 & 9.9 \\
\hline N.bw.R25 & 5233 & 1591 & 4104 & 23 & 25 & 74 & 4.7 & 211 & 0 & 11.6 \\
\hline N.bw.R56 & 9997 & 5237 & 31631 & 117 & 35 & 138 & 77.6 & 1125 & 0.1 & 11.3 \\
\hline N.bw.R58 & 8907 & 3696 & 22541 & 123 & 24 & 134 & 4.2 & 295 & 0 & 16 \\
\hline N.bw.R65 & 8535 & 1807 & 11246 & 18 & 16 & 84 & 4.6 & 166 & 0 & 12.5 \\
\hline N.bw.R4 & 7832 & 670 & 3638 & 10 & 39 & 35 & 1.6 & 103 & 0 & 11.6 \\
\hline N.bw.R39 & 7824 & 5399 & 12460 & 65 & 21 & 131 & 2.2 & 360 & 0 & 12 \\
\hline N.bw.R66 & 10213 & 6710 & 36041 & 331 & 47 & 192 & 32.8 & 1111 & 0 & 9.4 \\
\hline B.bw.1800.6 & 9929 & 2291 & 10674 & 63 & 100 & 94 & 3.1 & 188 & 0 & 12.4 \\
\hline B.bw.1830.1 & 9669 & 5426 & 19872 & 36 & 78 & 105 & 3.8 & 240 & 0 & 13.3 \\
\hline B.bw.1830.2 & 8954 & 3236 & 13708 & 16 & 75 & 72 & 2.2 & 231 & 0 & 11.1 \\
\hline B.bw.1830.3 & 7062 & 1572 & 6324 & 12 & 97 & 61 & 2 & 206 & 0 & 9.5 \\
\hline B.bw.1830.4 & 6559 & 3860 & 17031 & 28 & 98 & 49 & 3.1 & 218 & 0 & 13.2 \\
\hline B.bw.1850.1 & 9608 & 4799 & 17495 & 45 & 152 & 143 & 7.2 & 214 & 0 & 8.8 \\
\hline N.bw.R27 & 5620 & 2546 & 8396 & 170 & 26 & 123 & 436.8 & 115 & 0 & 7.2 \\
\hline N.bw.R36 & 7616 & 7192 & 24673 & 325 & 38 & 361 & 3 & 412 & 0.3 & 13.9 \\
\hline N.bw.R37 & 7089 & 3894 & 18134 & 73 & 50 & 83 & 2.3 & 277 & 0 & 10.5 \\
\hline N.bw.R63 & 6745 & 5123 & 23434 & 364 & 52 & 90 & 105.9 & 269 & 0.1 & 12.2 \\
\hline N.bw.R68 & 8238 & 8393 & 32481 & 303 & 29 & 178 & 6.7 & 679 & 0 & 8 \\
\hline B.bw.1890.1 & 4909 & 2062 & 9872 & 9 & 67 & 48 & 14.3 & 135 & 0.1 & 9.5 \\
\hline
\end{tabular}

QING Group 'B' 


$\begin{array}{ccccccccccc}\text { B.bw.1780.1a } & 6558 & 2294 & 10990 & 50 & 35 & 43 & 2.7 & 257 & 0 & 9 \\ \text { B.bw.1780.1b } & 4625 & 1872 & 8482 & 38 & 33 & 33 & 5.4 & 180 & 0 & 9.5 \\ \text { B.bw.1780.2a } & 8764 & 4885 & 24927 & 68 & 54 & 212 & 8 & 399 & 0 & 8.9 \\ \text { B.bw.1780.2b } & 11847 & 8600 & 39344 & 151 & 73 & 203 & 23.5 & 524 & 0 & 8.9 \\ \text { B.bw.1780.3 } & 7502 & 3750 & 16747 & 147 & 37 & 37 & 166.2 & 285 & 0 & 9.4 \\ \text { B.bw.1780.4 } & 9187 & 7114 & 34018 & 108 & 44 & 41 & 19.4 & 444 & 0 & 8.2 \\ \text { B.bw.1780.5 } & 6345 & 4328 & 19388 & 92 & 55 & 55 & 7.4 & 366 & 0 & 8.1 \\ \text { N.bw.R44 } & 9266 & 6544 & 31702 & 984 & 140 & 195 & 998.8 & 673 & 0 & 11.8 \\ \text { N.bw.R71 } & 4920 & 4703 & 18145 & 210 & 48 & 54 & 2.3 & 322 & 0 & 8.5 \\ \text { N.bw.R70 } & 6158 & 4626 & 24806 & 59 & 32 & 70 & 19.8 & 561 & 0 & 14.7\end{array}$

\section{QING Group ' $C$ '}

$\begin{array}{lcccccccccc}\text { B.bw.1800.1 } & 7921 & 1950 & 21738 & 139 & 34 & 83 & 30.1 & 1634 & 0 & 3.5 \\ \text { B.bw.1800.2 } & 8001 & 2256 & 24454 & 184 & 87 & 95 & 2 & 1541 & 0 & 3.9 \\ \text { B.bw.1800.3 } & 7645 & 1320 & 14007 & 13 & 11 & 83 & 0.8 & 1239 & 0 & 4.7 \\ \text { B.bw.1800.4 } & 3838 & 1272 & 10961 & 26 & 10 & 19 & 1.7 & 1216 & 0 & 2.2 \\ \text { B.bw.1800.5 } & 3803 & 572 & 5345 & 15 & 12 & 26 & 0.5 & 1025 & 0 & 1.9\end{array}$

\section{underglaze on \\ polychrome}

$\begin{array}{ccccccccccc}\text { V\&A.ub.C.176-1934 } & 8184 & 4214 & 24859 & 180 & 22 & 45 & 5.8 & 233 & 0 & 6.5 \\ \text { V\&A.ub.C.925-1921 } & 5290 & 301 & 3407 & 15 & 55 & 42 & 0.9 & 89 & 0 & 11.2 \\ \text { B.ub.1720.1 } & 10578 & 1220 & 12701 & 537 & 76 & 51 & 1.6 & 212 & 0.1 & 10.9\end{array}$

Table 5: LA-ICPMS analyses of blue enamels on famille verte and famille rose porcelains

\begin{tabular}{|c|c|c|c|c|c|c|c|c|c|c|c|c|}
\hline Sample & eign & Date & $\mathrm{Fe}$ & Co & Mn & $\mathbf{N i}$ & $\mathrm{Cu}$ & $\mathbf{Z n}$ & As & Ba & $\mathbf{B i}$ & $\mathbf{U}$ \\
\hline B.fv.1700.1 & . & 700 & 614 & 665 & 2 & 7 & 499 & 88 & 06 & 1 & 58 & 25 \\
\hline 1902 & noj & 1722 & 744 & 2283 & 5895 & 272 & 683 & 40 & 1848 & 136 & 364 & 12 \\
\hline B.fr. 1730.4 & Yor & 1730 & 6996 & 6519 & 1277 & 2397 & 328 & 1960 & 19847 & 8390 & 2729 & 0 \\
\hline B.fr. 1730.6 & Yongzheng & 1730 & 6979 & 4519 & 285 & 1662 & 429 & 2056 & 16340 & 3441 & 2012 & 179. \\
\hline B.fr. 1730.8 & Yongzheng & 1730 & 7741 & 4406 & 2611 & 1448 & 2074 & 1089 & 16162 & 956 & 2418 & 13. \\
\hline B.fr. 1730.9 & Yongrb & 1730 & 1736 & 5275 & 2779 & 1716 & 1805 & 1714 & 20882 & 3725 & 196 & 125.3 \\
\hline B.fr. 1740.1 & Qianlong & 1740 & 8844 & 8574 & 553 & 1736 & 357 & 1450 & 26158 & 5126 & 3505 & 51.3 \\
\hline B.fr. & 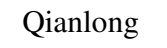 & 17 & 3 & 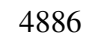 & 405 & 15 & 228 & 8 & 6 & 1223 & 574 & \\
\hline B.fr. 1750.3 & Qianlong & 1750 & 5405 & 5136 & 2673 & 1985 & 824 & 855 & 14613 & 313 & 11182 & 60.6 \\
\hline B.fr. 1770.1 & Qianlong & 1770 & 7602 & 4164 & 181 & 5883 & 529 & 184 & 12737 & 38 & 485 & 124.2 \\
\hline B.fr. 1770.3 & Qianlong & 1770 & 5620 & 4403 & 337 & 2157 & 305 & 846 & 8362 & 10741 & 993 & 93.1 \\
\hline B.fr. 1800.1 & Jiaqing & 1800 & 7411 & 5360 & 2329 & 1407 & 668 & 687 & 16012 & 829 & 1871 & 28.8 \\
\hline B.fr. 1850.1 & Daoguang & 1850 & 4099 & 13396 & 126 & 644 & 5613 & 450 & 22802 & 74 & 2073 & 3.9 \\
\hline B.fr. 1850.2 & Daoguang & 1850 & 2980 & 7516 & 271 & 490 & 293 & 138 & 18744 & 51 & 485 & 4.6 \\
\hline B.fr. 1850.3 & Daoguang & 1850 & 3671 & 2974 & 85 & 295 & 14785 & 371 & 15961 & 27 & 325 & 1.5 \\
\hline B.fr. 1850.4 & Daoguang & 1850 & 3792 & 5379 & 178 & 542 & 9482 & 1624 & 15713 & 136 & 1140 & 6.5 \\
\hline B.fr.1870.1 & Tongzhi & 1870 & 4849 & 20999 & 238 & 758 & 7567 & 583 & 33475 & 120 & 1533 & 5.1 \\
\hline
\end{tabular}


For the underglaze blue and white (Table 4), the main colouring element was, as expected, cobalt with an average of around 3300ppm Co. The blue and white underglaze blues have relatively low levels of iron (averaging 7650ppm Fe), raised levels of manganese, averaging 15,500ppm $\mathrm{Mn}$, and there is a clear correlation between the cobalt and manganese (Figure 1) and with zinc (Figure 2). These exceptionally high manganese contents render the contribution of manganese from the glaze (discussed above) as insignificant. There may also be correlations with barium (with some high barium outliers) and nickel, which is also elevated compared to the white glaze, but they are not as strong. The cobalt pigment from the Qing blue and whites looks very consistent, although it is interesting that the $\mathrm{Co} / \mathrm{Mn}$ ratio in the Qing $\mathrm{C}$ group is distinct from the others, being relatively richer in manganese, perhaps reflecting a slightly different source (see Figure 1 where the Co/Mn correlation is much steeper).

Figure 1: Plot of manganese against cobalt for underglaze blue painted areas on Qing blue and white Chinese porcelains and underglaze blues in polychrome wares

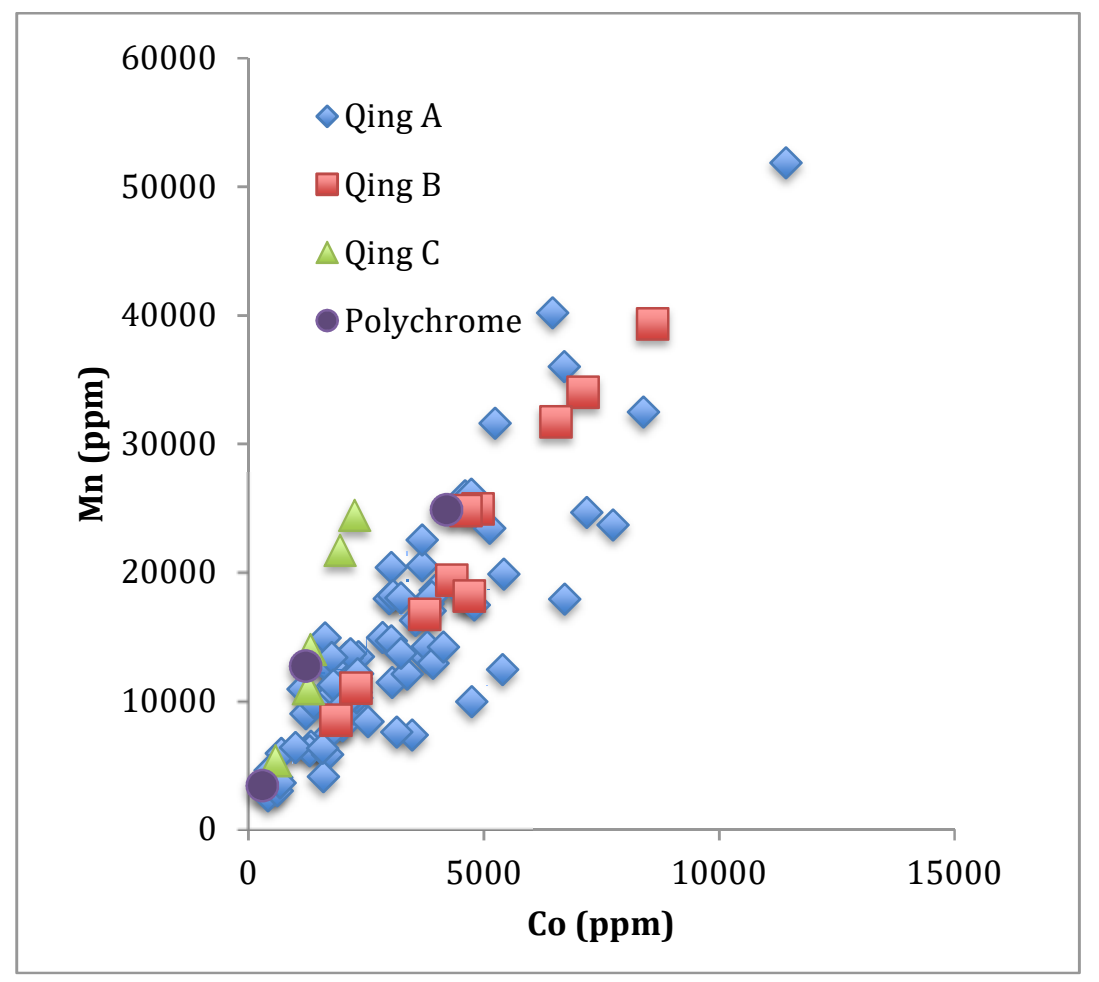

In contrast, there are two clear groups within the polychrome wares. Three of the analyses in Table 4 are underglaze blue from polychrome vessels (and plotted on Figure 1 and Figure 2 with the other underglaze blues). They are in many ways similar in composition to the underglaze blues of the blue and white (although one is higher in nickel). However the blue overglaze enamels on the polychrome wares are very distinct (Table 5). They are low in manganese, only $1240 \mathrm{ppm} \mathrm{Mn}$ on average, even though the cobalt concentrations are about twice those of the blue and white at 6300ppm. They are also higher in nickel (Figure 3) and zinc (Figure 4) and much higher in arsenic, bismuth and uranium (Figure 4 and Figure 5).. While this overglaze cobalt pigment is very variable, there is some separation between the enamels analysed according to date, with 
nineteenth century enamels having lower concentrations of other elements such as $\mathrm{Ni}$ and $\mathrm{U}$ relative to cobalt (Fig.6).

Figure 2: Plot of zinc against cobalt for blue glazes on Qing, blue and white Chinese porcelains and underglaze blues in polychrome wares

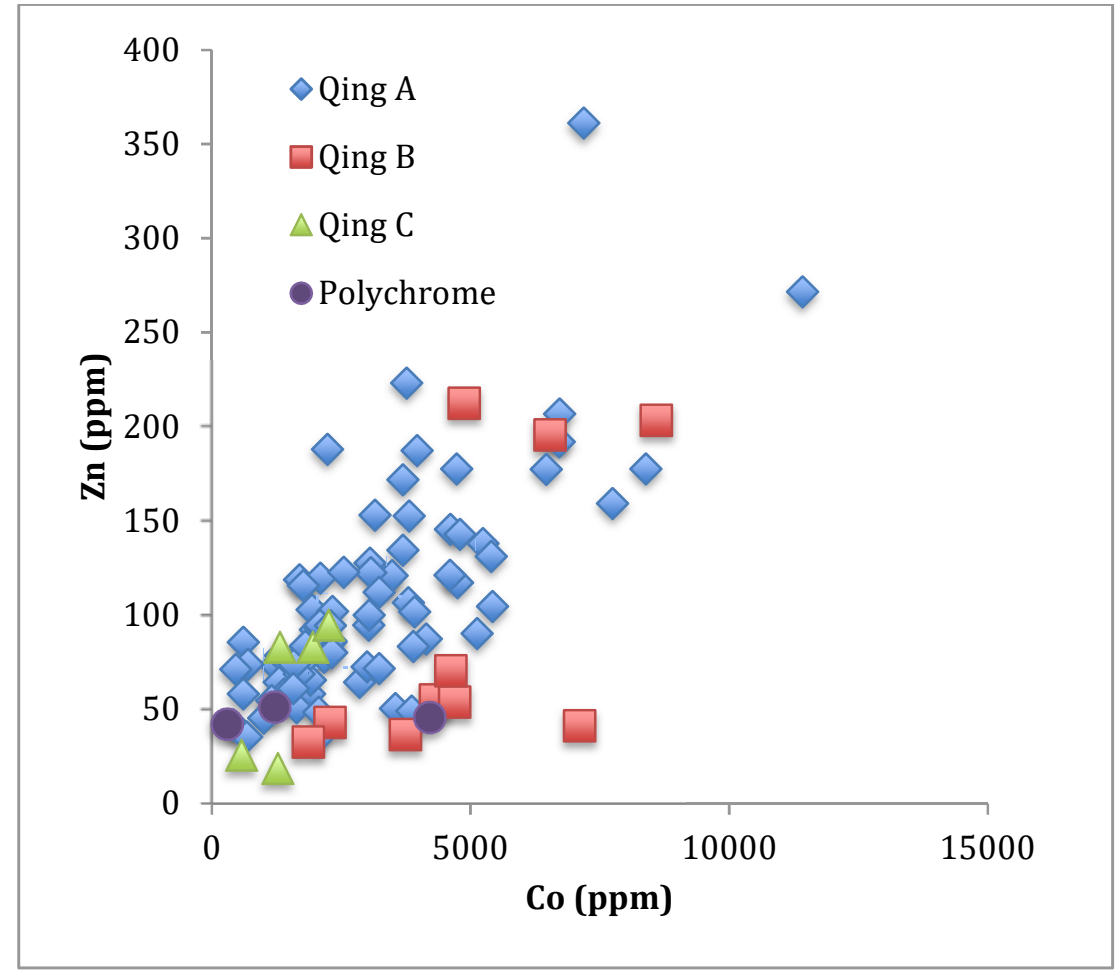

Figure 3: Plot of nickel against cobalt for blue enamels and underglazes of the polychrome wares

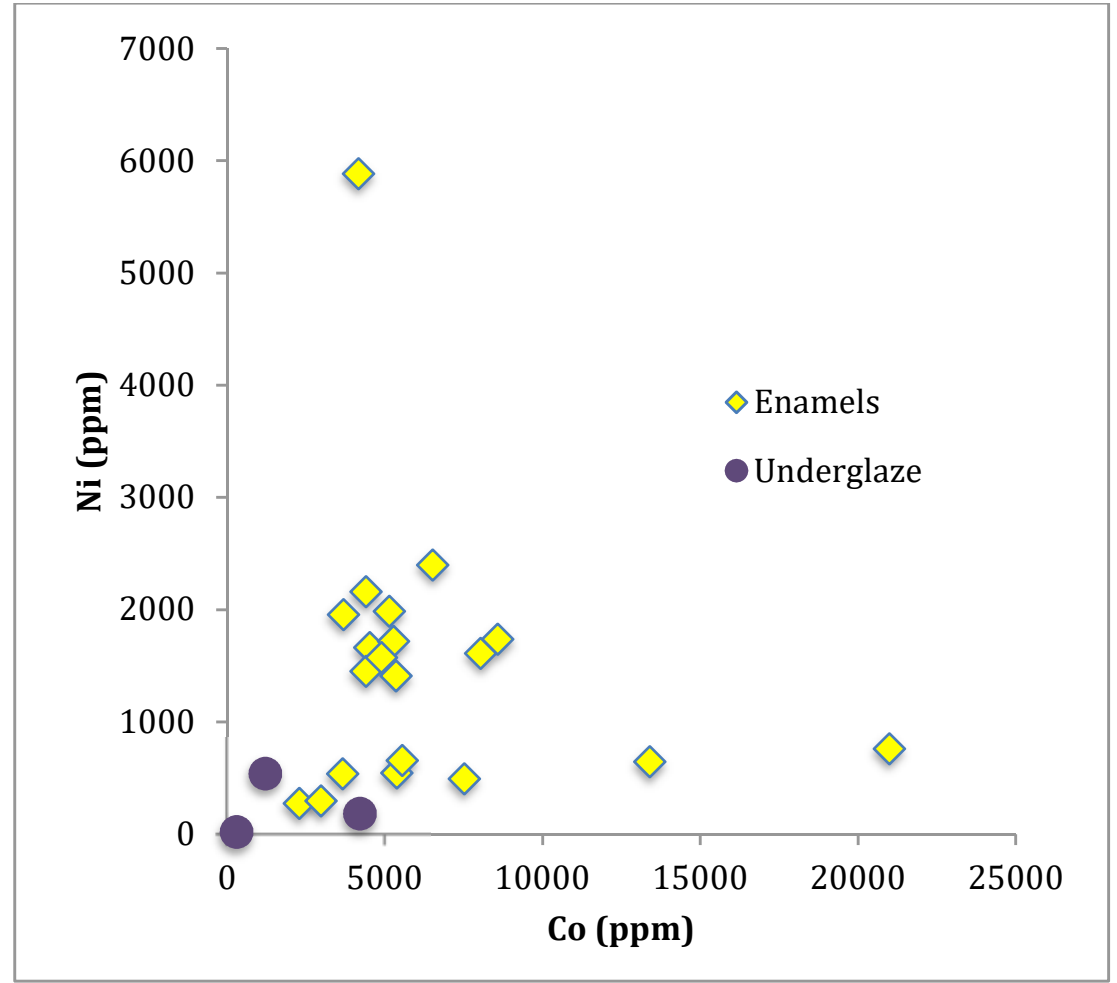


Figure 4: Plot of arsenic against zinc for blue enamels and underglazes of the polychrome wares

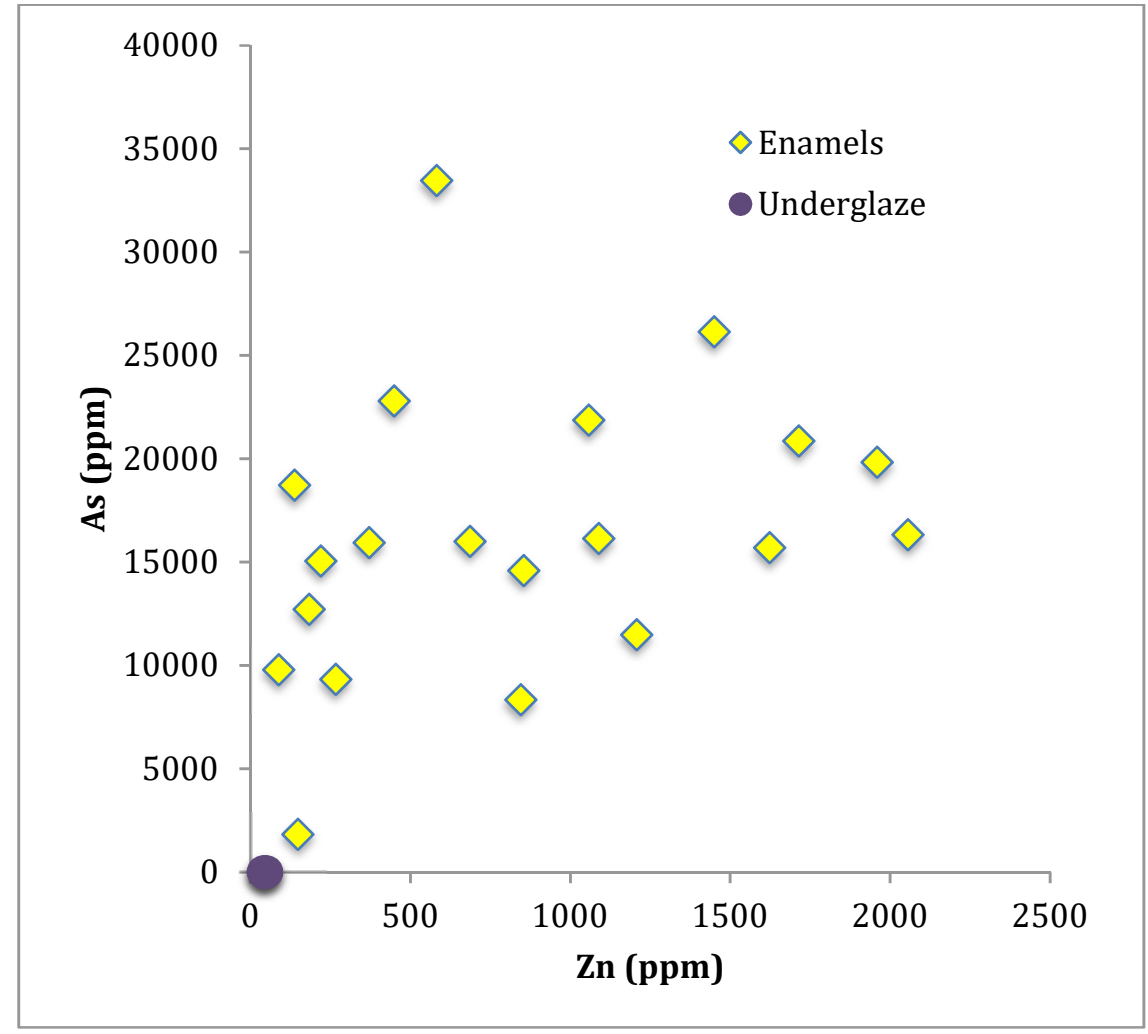

Figure 5: Plot of uranium against bismuth for blue enamels and underglazes of the polychrome wares

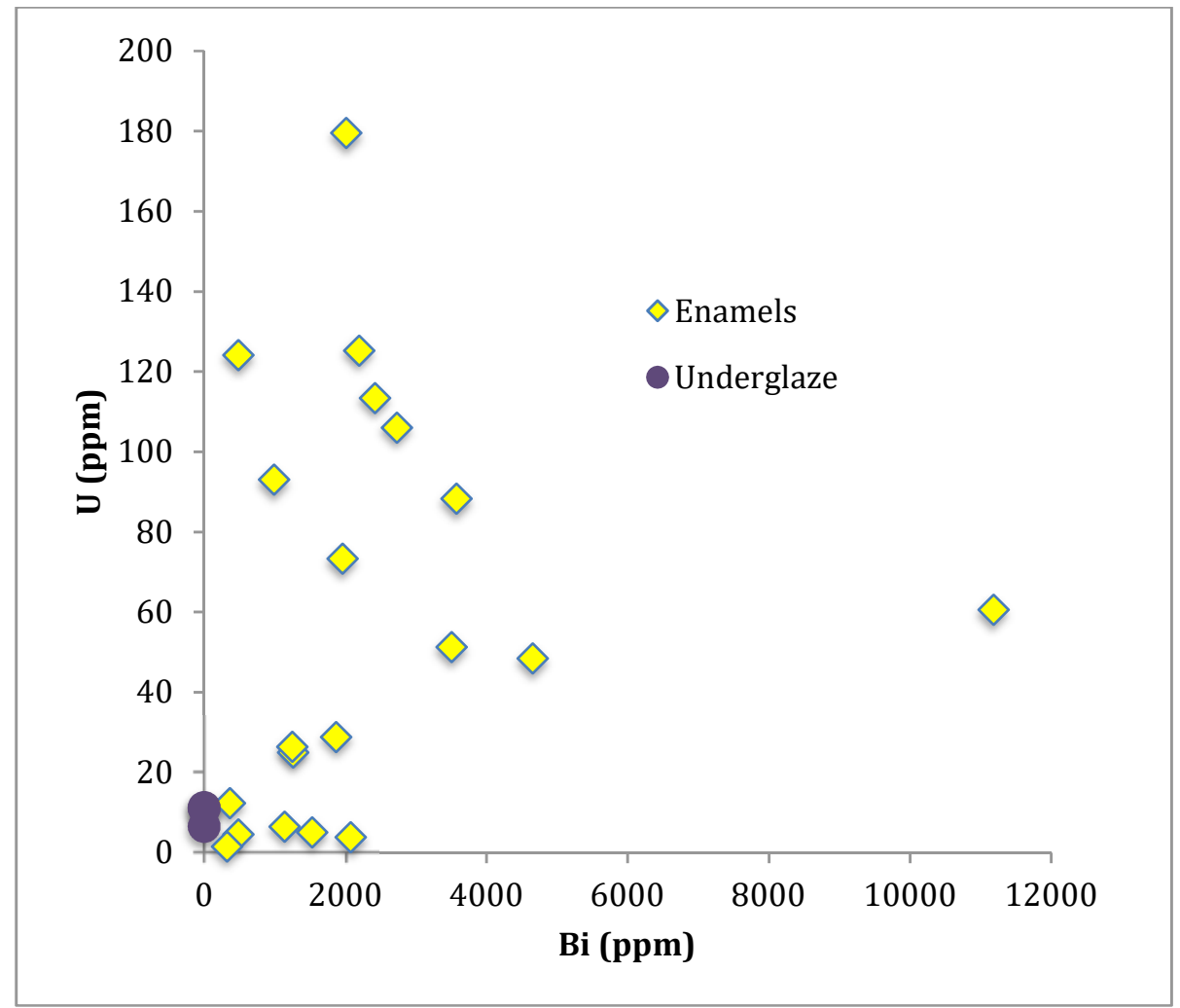


Figure 6: Ratios of cobalt to uranium and nickel in blue enamels according to date

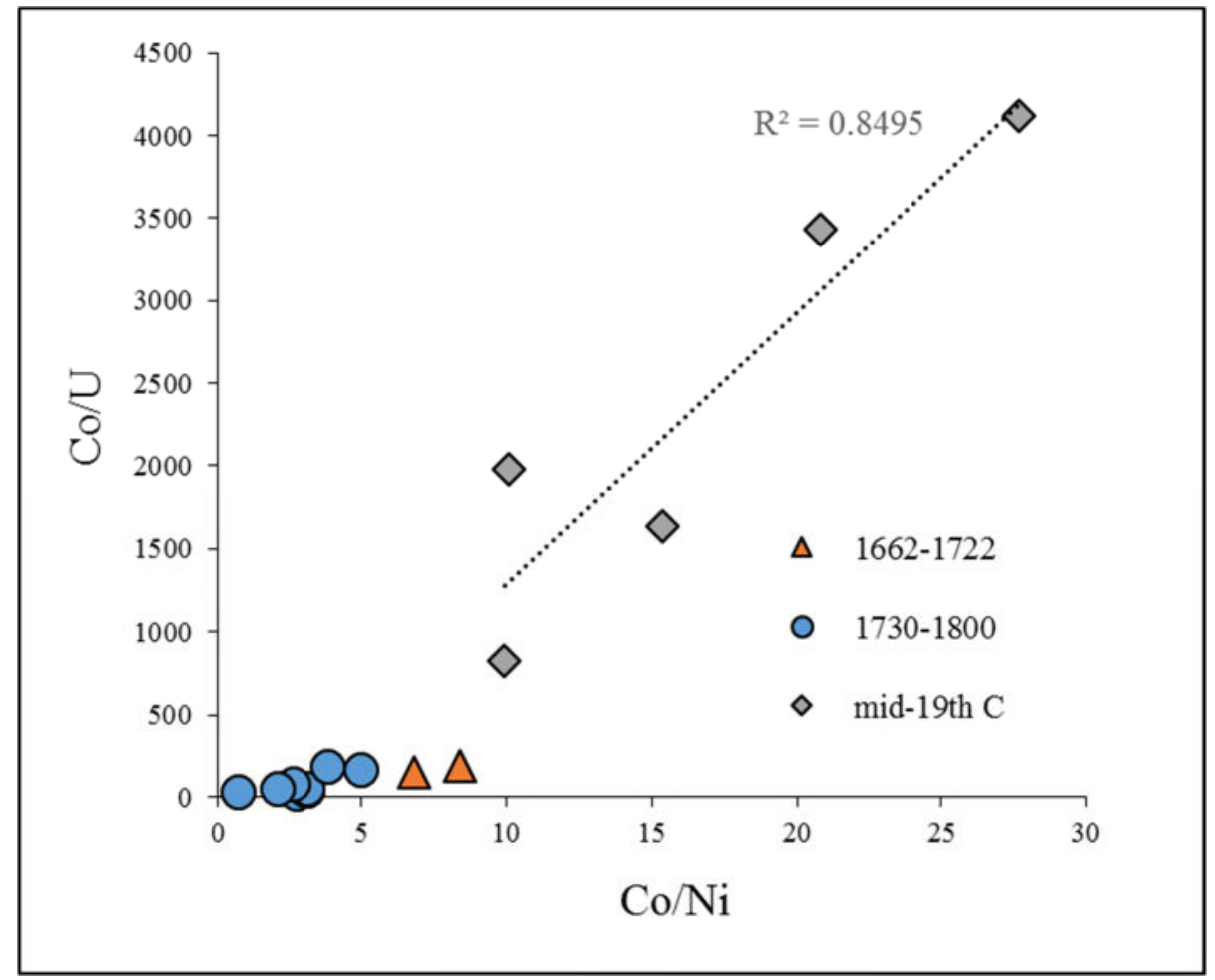

\section{Discussion}

All the analysed underglaze blue decorations of the Qing dynasty samples (Qing A-C) showed $\mathrm{Mn} /$ Co ratios that indicated the use of high Mn-cobalt pigments of the pyrolusite or "wad" type and C. However, there are some subtle differences between them. While Qing A and B groups have similar $\mathrm{Mn} / \mathrm{Co}$ ratios (average of $5.1 \pm 1.7$ and $4.7 \pm 0.4$ respectively, Qing $C$ is distinctly lower in Co, with a Mn/Co ratio of $10.1 \pm 1.1$. Qing $\mathrm{C}$ is also lower in $\mathrm{Ni}, \mathrm{Cu}$ and much lower in $\mathrm{As}$, but significantly higher in $\mathrm{Ba}$. There is the suggestion that the Qing B group might be bimodal with respect to Zinc (see Figure 2), three analyses having a Zn content of around $200 \mathrm{ppm}$, whereas the rest are only about $50 \mathrm{ppm}$. Although there are small variations, it seems reasonable to conclude that Qing A and B are perhaps from one Co source, whereas Qing C, while still of the high Mn pyrolusite type, may be from a different source. The results agree with analyses of blue glazes of both folk and imperial porcelain (recovered from the provinces of Jiangxi, Yunnan, Fujian, as well as from Hong Kong and spanning from the late Ming period until the end of the Qing dynasty), which revealed Mn/Co ratios mostly ranging between 4 and 8 (although the iron is at the lower end of those reported in previous analyses) (Yap \& Tang 1984; Yu \& Miao 1997; Yu \& Miao 1996; Yu \& Miao 1998; Cheng et al. 2004; Wen et al. 2007, Wen and Pollard 2016; Zhu et al 2016) . Nickel and zinc were the main pigment impurities. In terms of possible cobalt sources used for the underglazes, contemporaneous Chinese records stated that, at the time of the Qing dynasty, different cobalt pigments were obtained from the provinces of Zhejiang (e.g. possibly erythrite from the prefectures of Shaoxing and Jinhua, which included various subtypes, such as the yuanzhi, zhiliao, and tianqing), Yunnan, Jiangxi (Yunzhou and Fengcheng), 
Guangdong, and Guangxi. The ores from Zhejiang and Yunnan were considered of higher quality, while the Jiangxi cobalt pigment was superior to those of the Guangdong and Guangxi areas (Tichane, 1983, p. 201; Wang et al., 1993). In the early part of the $20^{\text {th }}$ century, it seems that the best blue was the chu-ming or chu-ming-liao from the province of Yunnan (or the pigment ti-lo, which was rated even above the chu-ming blue).

The cobalt pigment used in the dark-blue enamels is completely different. While it is quite variable, it is low in manganese (all but one is $<3000 \mathrm{ppm}$ ), ten times higher on average in nickel and eight times higher in zinc. The average arsenic values are very high, in excess of $16,000 \mathrm{ppm}$ As. While the bismuth in the underglaze samples never exceeds $1 \mathrm{ppm}$ and is often not detected, in the enamels it averages 2,300ppm. The uranium is also very different being significantly lower, only $10 \mathrm{ppm}$ on average compared to $60 \mathrm{ppm}$ in the enamels. It is clear that the enamels have a very different cobalt source to the proposed local source used in the underglaze blue on both the blue and white and the polychrome wares. No other examples of cobalt with this characteristic type of composition have been recorded in Chinese ceramics, although high nickel cobalt has been reported in Chinese Qing dynasty Jingdezhen enamel-type glazes (Wood et al 2002).

Gratuze (Gratuze, 2013 see especially Table 5.1.4) has a useful table based on and extension of his previous work (Gratuze et al., 1996, e.g. 1995) which lists nine groups of cobalt pigment groups used from the Middle Bronze Age to the nineteenth century AD in the West. Group 8 is listed as Co-As-Ni-Bi-W-Mo-U-Fe and is sourced to Erzgebirge in Germany. The source appears to be used as early as $1400 \mathrm{BC}$, and widely from the sixteenth century to eighteenth centuries AD and has been found in French glass (Soulier et al., 1996) and della Robbia glazed ceramics (Zucchiatti et al., 2006) amongst others. Of particular pertinence is its association with the production of eighteenth century English porcelain (Middleton and Cowell, 1993). It is one of the most important cobalt sources of the period and has a high arsenic, nickel and bismuth composition, with elevated uranium, very similar to that observed in the blue enamels of the famille rose porcelain. While it is not possible to fully exclude other possibilities on compositional grounds alone (for example Co-As-Ni ores occur in Iran and in some regions of China), the specific elemental signature observed here, notably the high $\mathrm{Bi}$ and $\mathrm{U}$, appears to have been associated only with early modern European cobalt to date and this coupled with the chronological coincidence in its use, and the use of a different underglaze cobalt in China, strongly argue that the cobalt pigment used in the Chinese enamels was imported from Europe.

This identification of Saxon cobalt on eighteenth century Chinese porcelain is consistent with the evidence uncovered by Watney (1973:1, footnote 6), who reported documentary evidence dating to 1778 and 1795 that the East India Company was exporting "smalts" to China from London, and which indicate that this trade was on such a scale that at least in 1795 this resulted in a shortage of material of the desired quality for porcelain manufacture in England. In fact, the data of the present study suggest that the export of cobalt from Europe to China for use in porcelain production continued throughout most of the eighteenth and 
into the nineteenth centuries. It is not clear if London was the only point of departure for Saxon cobalt. However, prodigious quantities of cobalt from continental Europe were imported into Britain for use not only in ceramics and glass, but also as a whitener in textile production. Watney (1973) notes that 286,739 pounds weight of smalt was imported into England in 1754. Re-export of a proportion of this material by the East India Company would have been logistically straightforward.

The compositional groupings within the $\mathrm{Ni}$ - and As- cobalts used in the enamels may reflect changes in production method (fig. 6). The strong correlation observed in the nineteenth century enamels may reflect the introduction of new practices to refine the ore (Copeland, 1980, pp. 162-3) and/or the sale of Saxon cobalt according to grade, i.e. the impurity content (Taylor 1977). However, it is noted that ceramic producers in nineteenth century Britain, for example at the Spode factory, favoured cobalt from Sweden, rather than Saxony (Copeland 1980) and it is possible that a change to cobalt derived from Swedish cobaltite (CoAsS) ores had occurred.

The apparently exclusive use of European cobalt in Chinese overglaze enamels requires explanation, particularly given that native cobalt blue pigment was being used in great abundance in the underglaze decoration of both enamelled and plain blue-and-white wares, so presumably was not in short supply. It seems likely that this was because the cobalt used in the overglaze enamels was in solution in the glass, rather than a crystalline pigment. The properties required of cobalt pigment are very different in enamel and underglaze decoration. In order to produce detailed decoration in underglaze blue it was necessary to immobilise the pigment under the glaze, so that sharp lines did not "bleed" and this had been mastered centuries before, using either an iron-rich imported cobalt pigment or, from as early as the Hogwe Period (late $14^{\text {th }}$ century) and abundantly later in the Ming Dynasty, native manganese-rich Chinese cobalt, which can also be relatively high in cobalt (Wen et al 2007, Wen and Pollard 2016). Stable underglaze painting would have been easier to achieve with cobalt applied in a crystalline form rather than as a glass. The development of a good enamel, with a pure blue colour and without discolouration would have been a very different problem. Overglaze enamels fluxed by lead, as are all of the Qing enamels (Kingery and Vandiver 1986) require firing in an oxidising kiln, otherwise the lead will tend to precipitate as metal or sulphide and blacken the glaze. Under such conditions the manganese in cobalt derived from Chinese asbolane-type ores would have tended to oxidise to give a counteracting purple colouration and a less pure blue, and the reddish tinge of Chinese cobalt blue is noted specialists such as Bushell (1896; 1981 edition p. 267). Indeed, this oxidisation of manganese is evidenced by the frequent use of manganese purple enamel in the famille rose palette. Saxon cobalt did not have this problem as the cobalt source was relatively low in Mn. Furthermore, the exported product was primarily in the form of "smalt" - a pre-prepared cobalt-bearing silicate glass fluxed with about $15 \% \mathrm{~K}_{2} \mathrm{O}$ which was widely used in frescoes and oil painting from the fifteenth century on (for analyses, see e.g. Ciliberto et al., 1994; Spring et al., 2005). In Europe, smalt was distinguished from "zaffre", the cobalt oxide pigment typically used in underglaze painting on ceramics. It seems likely that 
Chinese potters, who had mastered the production of a suitable underglaze pigment, had difficulty developing the desirable shade of blue enamel using locally available materials, so chose to use smalt from Saxony, which was the dominant cobalt in the European market. Interestingly, particles of European smalt have been identified in Qing Dynasty lacquer by Julie Chang (pers. comm.), confirming that this form of cobalt was being imported into China.

A potentially important finding is the identification of Saxon cobalt in the two examples of famille verte enamel with overglaze blue painting, which appear to date to the period of the Kangxi emperor (1662-1722; Table 5: B.fv.1700.1 and V\&A. fv.1385-1902). This is in the period in which the famille rose palette was developed in Beijing, before production had been taken up at Jingdezhen. In his second letter (1722) from Jingdezhen, Pere d'Entrecolles suggested that there was an opportunity to supply a good European cobalt into China (e.g. Burton, 1906, p. 121). The present analyses indicate, however, that this was already happening. It is quite possible that the traded cobalt was not reaching Jingdezhen, as it is known that some enamelling on ceramic was carried out in Beijing, and some in Canton, where there was an established industry of cloisonné enamelling on copper. Alternatively d'Entrecolles may have been unaware of the use of imported cobalt at Jingdezhen, in spite of the detailed report on craft practices that he provides. He indicates that Jingdezhen was said to have had a million inhabitants and three thousand furnaces, and it is unlikely that the full range of practices are represented in the letters. As reported by Kingery and Vandiver (1986) and also observed here, the blue enamel on famille verte has higher potash than the other colours, fully consistent with the use of potash-rich smalt as the colourant. However, it should also be noted that high potassium levels were a common characteristic of Chinese cloisonné enamels since the 15th century (Biron and Quette 1997, 35-40). It is possible therefore that relatively high potassium contents represent a Qing technology for producing glazes, so a combination of this and the smalt could be the reason for the higher potassium contents.

These findings also point to a clearer understanding of the use of the term "foreign colours" to describe the famille rose palette. The official list of patterns produced in Jingdezhen in the Yongzhen period (1723-35) refers to "foreign colours" or "foreign decoration" six times in a total of fifty-eight entries (Bushell, 1896; Hobson, 1948, p. 97). The meaning of this term has been unclear - was it due to the use of such a palette on European enamelled wares, was the colour technology based upon European practice, or were the enamels themselves imported? Attention has focused particularly upon the introduction of the gold pink enamel, as the use of gold-based reds and pinks became common in European glass and glaze at around the end of the seventeenth century, following the discovery by the German chemist Johann Kunckel that a finely divided precipitate of gold nanoparticles could be precipitated by tin to yield "Purple of Cassius" (Hunt 1976 for a detailed discussion). The discussion of the development of the famille rose palette has in particular focussed upon the use of colloidal gold pink and whether or not the pigment or the technology was directly imported from Europe but the evidence has been considered ambiguous (Kerr and Wood, 2004; Kingery and Vandiver, 1986) (Mills and Kerr 1999). In 
fact, the present study shows that the apparently simpler cobalt blue technology provides a direct link with European materials and emphasises a literal element in the use of the term "foreign colours".

\section{Conclusions}

The results of this study indicate that some Chinese cobalt sources of the pyrolusite-rich or wad type, for example Qing C, may be distinguished using compositional analysis. Furthermore it has been shown that the Ni- and Bi-rich pigments of the overglaze enamels of the later nineteenth century (Daoguang and Tongzhi, Table 5, Fig. 6) differ from the Yongzheng and Qianlong examples, suggesting a chronological change in the ore source or pigment processing. These findings have implications for the study of cobalt sources in general, as they indicate that quantitative analysis of the blue areas of blue-and-white glazes may provide information which is not only helpful in distinguishing productions of different ore deposit types, but may also discriminate on the basis of relatively minor variations in the pigment which occur due to production changes. The spatial resolution of LA-ICPMS, its sensitivity to concentrations at the ppm level and the quantification capabilities offer clear advantages over the less sophisticated versions of X-ray fluorescence, although these are obviously valuable in determining broad compositional groups.

This study of the blue pigments used in underglaze and enamelled Chinese porcelain has shown that there are two distinct pigments being used. While both are cobalt coloured, the elements associated with the cobalt are different. The underglaze blue on the blue and white porcelain and the polychrome underglaze blues have Mn/Co ratios that show a high Mn-cobalt pigment was used, with nickel and zinc as the main impurities. This is consistent with local, Chinese sources of cobalt. However, the enamels of the famille verte and famille rose wares are very different, having low manganese and much higher nickel, arsenic, bismuth and uranium.. This is unlike any cobalt source reported from other Chinese ceramics, but is very similar to a contemporary European source Erzgebirge in Germany. This was used in European ceramics and glass and textual sources have suggested that it was being imported into China. It is therefore very likely that the source of this pigment is Europe. While it has been suggested (but is unproven) that some of the technologies used in Qing porcelain enamels, for example colloidal pink, might be derived from Europe (Kerr and Wood 2004), this is the first evidence that a pigment itself was transported over large distances. It is possible that the pigment was in the form of smalt, a cobalt glass, which might explain why it was used for enamels, but not for underglaze blues but it is also likely to have provided a better shade of blue.

The blue enamels analysed here were exclusively made using European cobalt, and the trade in this material appears to have lasted throughout the eighteenth and nineteenth centuries. The chain of supply was extensive. Saxon cobalt was incorporated into a potassium silicate glass in Europe, then imported to European trading centres, including London, but possibly others. It was then traded on to Canton. From there it was taken up to Jingdezhen, where it was 
used to manufacture enamelled porcelain. The porcelain then travelled the reverse route, back to the consumers of Europe. This is an impressive early example of a globalised trading network. However, it is interesting to note that in its general form, if not its extent, it echoes the situation several centuries before, when Persian cobalt was used to decorate the blue and white wares of the Yuan Dynasty, which were then exported across the Islamic world.

\section{Acknowledgements}

We would like to acknowledge the help of Professor Nigel Wood and the Research Laboratory for Archaeology and the History of Art, University of Oxford for the kind loan of samples for this project. Our greatest thanks got to Colin Sheaf, of Bonhams Auctioneers, Bond Street who inspired the project and nurtured it with his insight and enthusiasm, in addition to providing important examples of wares for analysis. This project was funded jointly by Cranfield University and by Bonhams.

\section{References}

Biron, I. and Quette, B., 1997, Les premiers émaux chinois, Techne, 6, 35-40.

Burton, W., 1906. Porcelain- Its art and manufacture. Batsford, London.

Bushell, S.W., 1896. Oriental Ceramic Art, 1981 repri. ed. Frederik Muller.

Cheng, H.S., Zhang, Z.Q., Zhang, B., Yang, F.J., 2004. The non-destructive identification of early Chinese porcelain by PIXE, in: Nuclear Instruments and Methods in Physics Research, Section B: Beam Interactions with Materials and Atoms. pp. 16-19.

Ciliberto, E., Fragalà, I., Pennisi, G., Spoto, G., 1994. Bulk and surface characterization of early pigments. Case study of Renaissance smalt. Sci. Technol. Cult. Herit. 3, 163-8.

Copeland, R., 1980. Spode’s Willow Pattern. Studio Vista, London.

Curtis, E.B., 1993. European contributions to the Chinese glass of the early Qing period. J. Glass Stud. 35, 91-101.

Curtiss, E.B., 2009. Glass exchange between Europe and China, 1550-1800: diplomatic, mercantile and technological interactions. Ashgate, Farnham, England.

Frank, S., 1982. Glass and archaeology, No. 10. Academic Press, London.

Gratuze, B., 2013. Provenance Analysis of Glass Artefacts, in: Janssens, K (ed.), Modern Methods for Analysing Archaeological and Historical Glass, Volume I. pp. 311-343.

Gratuze, B., 1999. Obsidian characterisation by laser ablation ICPMS and its 
application to prehistoric trade in the Mediterranean and hte Nera East. J. Archaeol. Sci. 26, 869-891.

Gratuze, B., Soulier, I., Barrandon, J.N., Foy, D., 1995. The origin of cobalt blue pigments in French glass from the thirteenth to the eighteenth centuries, in: Trade and Discovery. British Museum Occasional Paper 109, London, pp. 123-33.

Gratuze, B., Soulier, I., Blet, M., Vallauri, L., 1996. De l'origine du cobalt: du verre à la céramique. Rev. d'archéométrie 77-94. doi:10.3406/arsci.1996.939

Henderson, J., 2000. The science and archaeology of materials. Routledge, London.

Henderson, J., 1989. The Technology of Sixteenth- and Seventeenth-Century Chinese Cloisonné Enamels. Archaeometry 2, 133-146.

Hobson, R.L., 1948. Handbook of the pottery and porcelain of the Far East, British Ms. ed. London.

Hunt, L.B., 1976. The true story of Purple of Cassius. Gold Bull. 9, 134-9.

Jochum, K.P., Weis, U., Stoll, B., Kuzmin, D., Yang, Q., Raczek, I., Jacob, D.E., Stracke, A., Birbaum, K., Frick, D.A., Günther, D., Enzweiler, J., 2011. Determination of reference values for NIST SRM 610-617 glasses following ISO guidelines. Geostand. Geoanalytical Res. 35, 397-429.

Kerr, R., Wood, N., 2004. Part XII: Ceramic Technology, in:Needham, J. (Ed.), Sceince and Civilisation Is China. Cambridge University Press, Cambridge.

Kingery, W.D., Vandiver, P.B., 1986. The eighteenth-century change in technology and style from the famille-verte palette to the famille-rose palette, in: Technology and Style: Proceedings of a Symposium on Ceramic History and Archaeology at the 87th Annual Meeting of the American Ceramic Society, 6 May 1985, Cincinnati. pp. 363-381.

Medley, 1989. The Chinese Potter: a practical history of Chinese ceramics, 3rd ed. London and New York.

Middleton, A.P., Cowell, M., 1993. Report on the examination and analysis of some porcelains from Longton Hall and West Pans. Post-Medieval Archaeol. 27, 94-108.

Mills, P., Kerr, R., 1999. A study of ruby-pink enamels on Chinese porcelain, with a comparison of Chinese pink glass and European pink enamels of ceramics, in: Jingkun, G. (Ed.), Proceedings of ISAC '99. Shanghai, pp. 258-65.

Rawson, J., 1984. Chinese ornament: the lotus and the dragon. Trustees of the British Museum by British Museum Publications, London. 
Sato, M., 1981. Chinese Ceramics, 1st English edition, New York and Tokyo.

Shortland, A.J., Rogers, N., Eremin, K., 2007. Trace element discriminants between Egyptian and Mesopotamian Late Bronze Age glasses. J. Archaeol. Sci. 34, 781-789.

Soulier, I., Gratuze, B., Barrandon, J.-N., 1996. The origin of cobalt blue pigments in French glass, in: Archaeometry 94: Proceedings of the 29th International Symposium on Archaeometry. pp. 133-140.

Spring, M., Higgitt, C., Saunders, D., 2005. Investigation of pigment-medium interaction processes in oil paint containing degraded smalt. Natl. Gall. Tech. Bull. 26, 56-70.

Tichane, R., 1983. Ching-Te-Chen: Views of a Porcelain City. The New York State Institute for Glaze Research, Painted Post, NY.

Vainker, S.J., 1989. Chinese pottery and porcelain. British Museum, London.

van Elteren, J.T., Tennent, N.H., Šelih, V.S., 2009. Multi-element quantification of ancient/historic glasses by laser ablation inductively coupled plasma mass spectrometry using sum normalization calibration. Anal. Chim. Acta 644, 19.

Vicenzi, E.P., Eggins, A., Logan, R., Wysoczanski, R., 2002. Microbeam characterisation of Corning archaeological reference glasses: new additions to the Smithsonian Microbeam standard collection. J. Res. Natl. Inst. Stand. Technol. 107, 719-727.

Wagner, B., Nowak, A., Bulska, E., Hametner, K., Günther, D., 2012. Critical assessment of the elemental composition of Corning archeological reference glasses by LA-ICP-MS. Anal. Bioanal. Chem. 402, 1667-1677.

Wang, Q., Chin, L., Xu, J., Wang, C., 1993. Underglaze blue and red: Elegant decoration on procelain of Yuan, Ming and Qing. Multi-Art, Hong Kong.

Wen, R., Pollard, A.M., 2016. The Pigments Applied to Islamic Minai Wares and the Correlation with Chinese Blue-and-White Porcelain. Archaeometry 58, $1-16$.

Wen, R., Wang, C.S., Mao, Z.W., Huang, Y.Y., Pollard, A.M., 2007. The chemical composition of blue pigment on Chinese blue-and-white porcelain of the Yuan and Ming dynasties (AD 1271-1644). Archaeometry 49, 101-115.

Weyl, W.A., 1951. Coloured glasses. Society of Glass Production, Sheffield.

Wood, N., 1999. Chinese Glazes: their origins, chemistry, and recreation. University of Pennsylvania Press, Philadelphia. 
Wood, N., Borgio, L., and Kerr, R., 2002, An evaluation of the composition and production processes of Chinese "Robin's Egg" glazes. Gu Taoci Kexue Jishu 5: ISAC '02. Shanghai, 337-353.

Yap, C.T., Tang, S.M., 1984. X-ray fluorescence analysis of modern and recent chinese porcelains. Archaeometry 26, 78-81.

Yu, I.N., Miao, J.M., 1996. Non-destructive analysis of Jingdezhen blue and white porcelains of the Ming Dynasty using EDXRF. X-ray Spectrom. 25, 281-285.

Yu, K.N., Miao, J.M., 1998. Multivariate analysis of the energy dispersive X-ray fluorescence results from blue and white Chinese porcelains. Archaeometry 40, 331-339.

Yu, K.N., Miao, J.M., 1997. Locating the origins of blue and white porcelains using EDXRF. Appl. Radiat. Isot. 48, 959-963.

Zhu, T., Ding, X., Kusimba, C.M., Feng, Z., 2015. Using laser ablation inductively coupled plasma mass spectroscopy (LA-ICP-MS) to determine the provenance of the cobalt pigment of Qinghua porcelain from Jingdezhen in Yuan Dynasty of China (1271-1368AD). Ceram. Int. 41, 9878-9884.

Zucchiatti, A., Bouquillon, A., Katona, I., D’Alessandro, A., 2006. The “della Robbia blue": A case study for the use of cobalt pigments in ceremics during the Italian Renaissance. Archaeometry 48, 131-152. 